\title{
Time expended on managing molar incisor hypomineralization in a pediatric dental clinic in Nigeria
}

\section{Morenike Oluwatoyin FOLAYAN ${ }^{(a)}$ Titus Ayodeji OYEDELE(b) Elizabeth OZIEGBE(a)}

(a) Obafemi Awolowo University, Department of Child Dental Health, Ile-Ife, Nigeria.

(b) Babcock University, Benjamin Carson (Snr) School of Medicine, Department of Surgey, Ilisan-Remo, Ogun State, Nigeria.

Declaration of Interests: The authors certify that they have no commercial or associative interest that represents a conflict of interest in connection with the manuscript.

Corresponding author: Morenike Oluwatoyin Folayan toyinukpong@yahoo.co.uk

Submitted: October 17, 2017

Accepted for publication: April 16, 2018

Last revision: June 19, 2018
Abstract: This study assessed the difference in the number of visits made to a dental care clinic and the time spent providing specific dental treatment for children with and without molar incisor hypomineralization (MIH). Children aged 8 to 16 years who presented at the Pediatric Dental Unit of the Obafemi Awolowo University Teaching Hospital Complex, in Ile-Ife, Nigeria, were eligible for the study. A comprehensive medical and dental history was taken, and each child was clinically examined, diagnosed, and treated according to a drawn-up plan. The time taken to establish a diagnosis and to provide specific treatments (scaling and polishing, restoration, pulpectomy, extraction, and placement of stainless steel crowns) and the number of visits made to complete the treatment plan were recorded for each child. Differences in the number of visits, time expended to make a diagnosis and to treat children with and without MIH were analyzed. The average time for diagnosis $(p=0.001)$ and the average time for placing amalgam restorations $(p=0.008)$ were significantly longer in children with MIH than in those without it. Children with MIH made more visits to the clinic $(p<0.001)$.There was no significant difference in the average time for scaling and polishing $(p=0.08)$, glass ionomer cement restorations $(p=0.99)$, composite restorations $(p=0.26)$, pulpectomy $(p=0.42)$, tooth extraction $(p=0.06)$, and placement of a stainless steel crown $(p=0.83)$ in children with and without MIH. In conclusion, children with $\mathrm{MIH}$ required more time for oral health care. Placing amalgam restorations took significantly longer than placing tooth bonding restorative materials in children with MIH than in those without it.

Keywords: Time; Tooth Demineralization; Children.

\section{Introduction}

Molar incisor hypomineralization (MIH) is a term used to describe a form of developmental defect of the enamel characterized as demarcated opacities involving one or all permanent molars with or without the involvement of the incisors. ${ }^{1,2}$ The number of hypomineralized first permanent molars in an individual can range from one to four. When MIH affects two or more first permanent molars, including the contralateral tooth, the lesion is described as moderate or severe. ${ }^{1,3,4}$ 
The prevalence of MIH ranges between 3.6\% and $40.0 \%{ }^{4}$ with a mean overall prevalence of $13.1 \%,{ }^{5}$ but Zhao et al. previously reported an overall prevalence of $14.2 \% .{ }^{6}$ Morbidities associated with MIH include caries and their sequelae, tooth sensitivity, and aesthetic concern with a possible negative impact on the quality of life of affected children. ${ }^{5,7,8}$ This implies that a number of children with MIH would need to seek dental treatment and have multiple dental visits over the course of their lifetime for the purpose of managing many complications associated with the lesion - post-eruptive breakdown, pulp diseases, hypersensitivity, or pain. ${ }^{5}$ For children who need to make multiple dental visits, the management of $\mathrm{MIH}$ may significantly affect school time. ${ }^{9}$ Morbidities associated with MIH may also affect growth and development significantly. ${ }^{10}$ By the age of 9 years, children with MIH will have undergone dental treatment of their first permanent molars almost 10 times more than their peers. They also have an 11-fold higher probability of requiring restorative treatment of their first permanent molars compared with children without MIH..$^{11,12}$

In Nigeria, a household-based study showed that the prevalence of $\mathrm{MIH}$ was $9.7 \%{ }^{13}$ while a school-based survey revealed a prevalence rate of $17.7 \%{ }^{4} \mathrm{~A}$ large number of children may, therefore, need to visit a dental clinic for preventive, restorative, or orthodontic care, or tooth extractions for management of MIH. ${ }^{5}$ For the sake of treatment planning, it is therefore important to assess the number of dental visits that may be required for the management of a patient with MIH as well as the clinical time expended managing these children. This study tried to address both of these concerns.

\section{Methodology}

The study recruited children aged 8 to 16 years enrolled in a school screening program who presented to the Pediatric Dental Unit of the Department of Child Dental Health at Obafemi Awolowo University Teaching Hospital Complex. The recruitment took place over a 12-month period. Details of the screening program were previously published by Oyedele et al. ${ }^{7}$ All children diagnosed with $\mathrm{MIH}$ and those without
MIH but who also needed dental treatment during the school screening program were invited for free oral health care.

This study recruited children who were visiting the dental clinic for the first time. This decision was made in order to exclude children who might have dental phobia due to a previous negative experience in a dental clinic. ${ }^{14}$ Also, prior exposure to dental care may affect dental chair behavior, which may impact on the time expended on dental treatment. In addition, children who were attending the dental clinic for the first time but who required pharmacological management of their behavior or of dental anxiety were excluded from the study for the same reason.

All children recruited for the study had comprehensive history taken at the first dental visit using a standard clerking format. Information obtained during the history taken included age, sex, socioeconomic status, presenting complaint, history of presenting complaint, past and current medical history, past dental history, family history, and social history.

The socioeconomic status of each child was classified as class I (upper class), class II (upper middle class), class III (middle class), class IV (lower middle class), or class $\mathrm{V}$ (lower class). ${ }^{15}$ For ease of analysis, socioeconomic status in this study was regrouped into three levels: high (upper and upper middle classes), middle (middle class), and low (lower middle and lower classes). ${ }^{16}$

A systematic review was also conducted. Detailed clinical examination was also performed, which consisted of general physical examination, extra-oral examination including an assessment of the facial profile, intraoral examination of the intra-arch and inter-arch dental relationships, and examination of the oral mucosa and soft tissues. When necessary, children could be subjected to radiographs and tooth sensitivity tests. For each child, a definitive diagnosis was made after adequate investigation. A treatment plan was then drawn up.

For children with MIH, a clinical examination was conducted to determine the severity of the lesion using the criteria established in earlier publications. ${ }^{17,18}$ Children were diagnosed with MIH when at least one molar was affected, with or without involvement of the incisors. Each first permanent molar and incisor was screened for demarcated white, yellow, or brown 
opacities greater than or equal to $2 \mathrm{~mm}$ in diameter. Severity of the lesions was classified as mild MIH if the tooth had demarcated opacities with no need of treatment; moderate MIH if the lesions on the tooth had rough and broken down enamel alone; and severe if the hypomineralized lesion was associated with loss of dental structure affecting both enamel and dentin, with atypical restorations replacing affected hard tissue. Each child's hypomineralization grade was defined by the most severe defect seen in his/ her first permanent molars or permanent incisors.

The time spent by the dentists on history taking and clinical examination was recorded. The time spent on managing patients for any of the following procedures was also recorded: scaling and polishing, restoration of carious lesions using glass ionomer cement (GIC), composite or amalgam, pulpectomy, extraction, or placement of stainless steel crown (SSC). The time spent on providing each patient with any of the treatment procedures during a single dental visit when indicated in the treatment plan was recorded. The number of visits made to the clinic for the management of dental lesions diagnosed during the first dental visit was also recorded. The time required for diagnosis and treatment as defined by Ligali et al. ${ }^{19}$ was adopted for this study.

The time required for history taking was defined as the period during which a thorough history of the patient was taken. It commenced from the time the patient sat in the dental chair and ended when history taking had been completed. Examination time was defined as the period during which extra-oral and intra-oral examinations were conducted while the patient was sitting in the dental chair. For the purpose of this study, the time required to make a diagnosis, herein referred to as diagnostic time, is the total time expended on taking a history, conducting a clinical examination, undertaking investigations, and drawing up the treatment plan.

Treatment time was defined as the period during which each lesion received actual treatment during the dental visit. It was measured from the beginning of treatment to the last visit. For the study, the time spent on scaling and polishing, placement of restorations (amalgam, GIC, and composite), pulpectomy, extraction, and SSC placement was recorded.

Data analysis was conducted using STATA version 11. Descriptive analysis was performed. Differences in the time expended making a diagnosis and treating lesions and the number of follow-up visits for management of lesions diagnosed during the first dental visit between patients with and without MIH were analyzed using t-tests, and chi-square tests where appropriate. Statistical differences were inferred when the $\mathrm{p}$ value was equal to or less than 0.05 .

The study was approved by the Research Ethics Committee (ERC/2011/06/03) of the Obafemi Awolowo University Teaching Hospital Complex. Permission was also obtained from the Nigerian Ministry of Education and from the heads of all schools that participated in the school screening program. Parental consent was received for all children who participated in the study. All data were handled confidentially. Children were clerked with parents present in the dental cubicle. However, for the sake of privacy, adolescents aged 12 to 16 years were clerked without their parents in the dental cubicle. All treatments were undertaken in the presence of parents in the dental cubicle.

The school screening program and the clinical management of patients were conducted by one of the authors (TAO). A standard operating procedure was developed for the entire study. The weighted kappa statistic was used to determine the intra-examiner reliability for the diagnosis of MIH. The score was 0.85 .

\section{Results}

A total of 2,107 schoolchildren aged 8 to 16 years were screened. Of these, 267 were diagnosed with $\mathrm{MIH}$ and 395 were diagnosed with other oral lesions exclusive of MIH.

Of the 267 children diagnosed with MIH, only 41 (15.4\%) met the inclusion criteria. All of the 41 children were recruited for the study. Of the 395 children without MIH who had other oral lesions, 32 (8.1\%) met the inclusion criteria. They were all recruited for the study and served as the comparison arm for the study. Significantly more children with MIH showed up for clinical care $(p=0.003)$.There was no significant difference in the proportion of referred patients with $\mathrm{MIH}$ and those referred without MIH who showed up for clinical care based on sex $(p=0.21)$, age $(p=$ 0.23 ), and socioeconomic status $(p=0.15)$ (Table 1$)$. 
Time expended on managing molar incisor hypomineralization in a pediatric dental clinic in Nigeria

Table 1. Sociodemographic variables of patients who presented for clinical care.

\begin{tabular}{|c|c|c|c|c|}
\hline \multirow{3}{*}{ Variables } & \multicolumn{2}{|c|}{ Molar incisor hypomineralization } & \multirow{2}{*}{ Total } & \multirow{3}{*}{$\mathrm{p}$-value } \\
\hline & Present & Absent & & \\
\hline & $(n=41)$ & $(n=32)$ & $n=73$ & \\
\hline Mean age & $11.61+(2.61)$ & $10.91+(2.29)$ & $11.30+(2.48)$ & 0.23 \\
\hline \multicolumn{5}{|l|}{ Sex } \\
\hline Male & $24(58.5 \%)$ & $14(43.8 \%)$ & $38(52.1 \%)$ & \\
\hline Female & $17(41.5 \%)$ & $18(56.2 \%)$ & $35(47.9 \%)$ & 0.21 \\
\hline \multicolumn{5}{|c|}{ Socioeconomic status } \\
\hline High & $6(14.6 \%)$ & $9(28.1 \%)$ & $15(20.5 \%)$ & \\
\hline Middle & $17(41.5 \%)$ & 7 (21.9\%) & $24(32.9 \%)$ & 0.15 \\
\hline Low & $18(43.9 \%)$ & $16(50.0 \%)$ & $34(46.6 \%)$ & \\
\hline
\end{tabular}

The average diagnostic time was $85.86+(35.01)$ minutes. There was a significant difference in the average diagnostic time between children with and without MIH: the average diagnostic time was longer for children with MIH when compared with those without MIH (97.43 minutes vs. 71.03 minutes; $\mathrm{p}=0.001$ ).

Table 2 shows the average time for specific treatments for children diagnosed with $\mathrm{MIH}$ and those without MIH. Sixty-six children were subjected to scaling and polishing: 28 (42.4\%) children without $\mathrm{MIH}$ and 38 (57.6\%) with MIH. Though the average time for scaling and polishing for children with $\mathrm{MIH}$ was longer than that for children without MIH, there was no significant difference in the time (29.76 minutes vs. 34.39 minutes; $p=0.08$ ).

Thirty-two children had GIC restorations: 18 (56.3\%) children with $\mathrm{MIH}$ and 14 (43.7\%) without MIH. There was no significant difference in the average time for placement of a GIC restoration for children with MIH and children without MIH (37.42 minutes vs. 37.39 minutes; $p=0.99$ ).

Twenty-three children had amalgam restorations: eight (34.8\%) children with MIH and 15 (63.2\%) without $\mathrm{MIH}$. There was a significant difference in the average time for placement of an amalgam restoration for children with $\mathrm{MIH}$ and children without $\mathrm{MIH}$ : the time for placement of an amalgam restoration for children with MIH was longer than that for children without MIH (66.13 minutes vs. 41.2minutes; $p=0.008$ ).

Thirteen children had composite restorations: six $(46.2 \%)$ children with MIH and seven (53.7\%) without MIH. There was no significant difference in the average time for placement of a composite restoration for children with and without MIH (46.14 minutes vs. 64.17 minutes; $p=0.26$ ).

Seventeen children had pulpectomy: 15 (88.2\%) children with MIH and two (11.8\%) without MIH. Though the average time for pulpectomy for children with MIH was longer than that for children without $\mathrm{MIH}$, there was no significant difference in the time (142.87 minutes vs. 94.5 minutes; $\mathrm{p}=0.42$ ).

Thirty-four children needed a tooth extraction: 29 (85.3\%) children with MIH and five (14.7\%) without $\mathrm{MIH}$. Though the average time for tooth extraction for children with $\mathrm{MIH}$ was longer than that for children without $\mathrm{MIH}$, there was no significant difference in the time (43.28 minutes vs. 21.4 minutes; $p=0.06$ ).

Seven children needed SSC placement: two (28.6\%) children with $\mathrm{MIH}$ and five (71.4\%) without $\mathrm{MIH}$. There was no significant difference in the time for SSC placement for children with MIH when compared with those without MIH (115.5 minutes vs. 110.0 minutes; $p=0.83$ ).

On average, a child with MIH made significantly more visits to the dental clinic than a child without MIH (4.0 vs. 2.29; $\mathrm{p}<0.001)$. Table 3 provides the details of the average time required for specific treatments for children diagnosed with MIH based on the severity of the lesion. The average diagnostic time for children with mild, moderate, and severe MIH was 99.0 minutes, 81.5 minutes, and 100.32 minutes, respectively. When the average diagnostic time for children without MIH was compared with that for children with mild, moderate, 
and severe $\mathrm{MIH}$, respectively, there was no significant difference in the average diagnostic time for children with mild MIH (99 minutes vs. 71.03 minutes; $p=0.08$ ) and moderate MIH (81.5 minutes vs. 71.03 minutes; $p=0.49$ ). The difference in the average diagnostic time for children with severe MIH was significantly higher than that for children without MIH (100.32 minutes vs. 71.03 minutes; $p=0.0005$ ).

The average time for placement of an amalgam restoration, a pulpectomy, and a tooth extraction increased with the severity of $\mathrm{MIH}$, while the average time for a composite restoration decreased as the severity of MIH increased.

\section{Discussion}

The study showed that the average time for the diagnosis and treatment of children is significantly affected by MIH. It takes significantly longer to establish a diagnosis for children with MIH than for those without MIH. The diagnostic time for children with mild and moderate MIH was non-significantly longer when compared with children without MIH, and significantly longer for children with severe MIH when compared with children without MIH. It also takes significantly longer to place an amalgam restoration for children with $\mathrm{MIH}$ when compared

Table 2. Average clinical times and average number of dental visits for management of children with and without molar incisor hypomineralization.

\begin{tabular}{|c|c|c|c|c|}
\hline \multirow{3}{*}{ Variables } & \multicolumn{2}{|c|}{ Molar incisor hypomineralization } & Total & \multirow{3}{*}{$p$-value } \\
\hline & \multicolumn{3}{|c|}{ Mean time $+(S D)$} & \\
\hline & Present $(n=41)$ & Absent $(n=32)$ & $n=73$ & \\
\hline Average diagnostic time in minutes & $97.43+(34.0)$ & $71.03+(30.85)$ & $85.86+(35.01)$ & 0.001 \\
\hline \multicolumn{5}{|c|}{ Average specific treatment time in minutes } \\
\hline Scaling \& polishing & $29.76+(10.16)$ & $34.39+(10.82)$ & $31.72+(10.62)$ & 0.08 \\
\hline Amalgam filling & $41.2+(14.31)$ & $66.13+(26.88)$ & $49.89+(22.53)$ & 0.008 \\
\hline Composite restoration & $46.14+(24.05)$ & $64.17+(30.56)$ & $54.46+(27.67)$ & 0.26 \\
\hline GIC restoration & $37.43+(10.61)$ & $37.39+(5.73)$ & $37.41+(13.52)$ & 0.99 \\
\hline Pulpectomy & $142.87+(80.0)$ & $94.5+(6.36)$ & $137.18+(76.55)$ & 0.42 \\
\hline Extraction & $43.28+(24.64)$ & $21.4+(2.07)$ & $40.05+(24.04)$ & 0.06 \\
\hline Stainless steel crowns & $115.8+(34.20)$ & $110.0+(7.07)$ & $114.14+(28.22)$ & 0.83 \\
\hline Average number of dental visits & $4.0+(1.20)$ & $2.29+(0.64)$ & $3.26+(1.31)$ & $<0.001$ \\
\hline
\end{tabular}

Table 3. Average clinical times and average number of dental visits for management of children with different levels of molar incisor hypomineralization severity

\begin{tabular}{|c|c|c|c|}
\hline \multirow{3}{*}{ Variables } & \multicolumn{3}{|c|}{ Molar incisor hypomineralization } \\
\hline & \multicolumn{3}{|c|}{ Mean time $+(S D)$} \\
\hline & Mild $(n=4)$ & Moderate $(n=6)$ & Severe $(n=31)$ \\
\hline Average diagnostic time in minutes & $99.0+(13.0)$ & $81.5+(49.39)$ & $100.32+(32.53)$ \\
\hline \multicolumn{4}{|c|}{ Average specific treatment time in minutes } \\
\hline Scaling \& polishing & $30.25+(10.81)$ & $36.67+(8.19)$ & $28.21+(10.15)$ \\
\hline Amalgam filling & $46.0+(0.0)$ & $60.0+(0.0)$ & $70.5+(30.01)$ \\
\hline Composite restoration & $90.0+(0.0)$ & $50.0+(0.0)$ & $36.6+(16.40)$ \\
\hline GIC restoration & $37.0+(0.0)$ & $43.33+(4.16)$ & $30.14+(17.64)$ \\
\hline Pulpectomy & $79.0+(0.0)$ & $133.25+(55.46)$ & $153.1+(91.20)$ \\
\hline Extraction & $34.33+(11.59)$ & $34.75+(12.37)$ & $46.05+(27.24)$ \\
\hline
\end{tabular}


with children without MIH. Children with MIH make significantly more visits to a dental clinic than those without MIH.

One of the strengths of the study is the recruitment of patients who were attending the clinic for the first time. This reduces the potential influence of dental phobia on the time required for clinical care and also reduces the bias that familiarity with the dental clinic could have had towards diagnostic time. Also, by using first-time visit to the dental clinic, there was no risk of recruiting children who have been emotionally overloaded with dental care, which could lead to behavioral problems during dental treatment. ${ }^{11}$

However, there are other factors that could affect the duration of patient care such as anxiety, challenges with behavior management, clinician's competency, and availability of appropriate tools for clinical care. The study tried to limit the influence of these variables by ensuring that clinical care was provided by a single competent clinician. Also, children recruited for the study had reached the age at which their behavior could be effectively managed using nonpharmacological techniques. Having a clinician who has the competency to diagnose MIH also reduces the challenges associated with establishing the diagnosis of MIH. However, the study was not blinded. This increased the risk of the clinician influencing the result of the study. The small sample size and the recruitment of a convenience sample of study participants who showed up for dental treatment following referral also increased the risk for spurious findings during the sub-analysis.

Despite these limitations, the study is important for a number of reasons. First, it provides clinicians with information that can help them plan the patient's treatment time. The information can also help clinicians make critical decisions on how to optimize the limited time available for patient's treatment. Second, it also provides evidence for promoting the use of tooth bonding restorative materials for $\mathrm{MIH}$ rather than amalgam restoration when a decision about time needs to be made.

Treatment of MIH can be demanding and timeconsuming. Time is required for achieving adequate pain control, managing dental phobia, and controlling behavioral problems. It is equally important to determine the appropriate restorative material and optimally prepare the tooth to receive the material. ${ }^{20}$ This challenge was posed by amalgam restorations. The longer time required for amalgam restoration in children with MIH may result from challenges associated with the preparation of mechanical retention features for amalgam restoration of $\mathrm{MIH}$-affected teeth, which is not required for GIC and composite restoration of $\mathrm{MIH}$-affected teeth. Also, the difficulties encountered in providing $\mathrm{MIH}$-affected teeth with adequate anesthesia may increase the time necessary for amalgam restorations of such teeth. ${ }^{21}$ In addition, $\mathrm{MIH}$-affected teeth are hypersensitive to mechanical manipulation as a result of constant pulpinflammation. ${ }^{21}$

This finding does not imply support for the use of GIC and/or composite restorations for the management of MIH-affected molars in the long term. Hypomineralized enamel reduces the modulus of elasticity and is less resistant to abrasion, thereby increasing the prospect of loss of restorative materials. ${ }^{22}$ The use of SSC to restore MIH-affected teeth reduces the chances of possible loss of restorative materials and the long time required for placement of an amalgam restoration. This study shows that there is no significant difference in the time expended on placing an SSC in MIH-affected and non-MIH-affected teeth, though more time is required for placing an SSC when compared to placing restorative materials. The durability of SSC may compensate for the time expended in the clinic in the long run.

Composite resin is the recommended restorative material for fully erupted $\mathrm{MIH}$-affected teeth. ${ }^{23}$ However, MIH-affected enamel is less amenable to acid etching, making the retention of composite resins challenging. ${ }^{20}$ Also, there might be the need to place retention features on the tooth as the severity of MIH increases. ${ }^{24}$ Our finding that composite restoration requires less time as the severity of $\mathrm{MIH}$ increases is contrary to conventional expectations and difficult to explain. A plausible explanation provided by Kopperud et al. ${ }^{20}$ is that these patients may be so challenging to treat that dentists limit tooth substance removal. There was no other study we could compare our findings with. In view of the importance of composite resins for the restoration of MIH-affected teeth, it is important to further 
investigate this finding to be able to validate it and also to find the reason(s) for the observation.

Previous studies had demonstrated the high number of visits to dental clinics made by children with MIH. ${ }^{12}$ This study further highlights that children with MIH-affected teeth make more clinical visits for oral health care than those without MIH. As pointed out by Oyedele et al., the high incidence of co-morbidities associated with MIH may be one of the reasons why children with MIH-affected teeth make more dental visits. The second one is that the long procedural time for children with MIH implies that fewer procedures can be performed during each visit. These factors have far-reaching implications. Previous studies had shown that MIH impacts negatively on the quality of life of affected children. ${ }^{11}$ School absenteeism and the high number of dental

\section{References}

1. Weerheijm KL, Jälevik B, Alaluusua S. Molar-incisor hypomineralisation. Caries Res. 2001 Sep-Oct;35(5):390-1. https://doi.org/10.1159/000047479

2. Lygidakis NA, Dimou G, Marinou D. Molar-incisorhypomineralisation (MIH). A retrospective clinical study in Greek children. II. Possible medical aetiological factors. Eur Arch Paediatr Dent. 2008 Dec;9(4):207-17. https://doi.org/10.1007/BF03262637

3. Alaluusua S, Lukinmaa PL, Koskimies M, Pirinen S, Hölttä P, Kallio M et al. Developmental dental defects associated with long breast feeding. Eur J Oral Sci. 1996 Oct-Dec;104(5-6):493-7. https://doi. org/10.1111/j.1600-0722.1996.tb00131.x

4. Oyedele TA, Folayan MO, Adekoya-Sofowora CA, Oziegbe EO, Esan TA. Prevalence, pattern and severity of molar incisor hypomineralisation in 8- to 10-year-old school children in IleIfe, Nigeria. Eur Arch Paediatr Dent. 2015 Jun;16(3):277-82. https://doi.org/10.1007/s40368-015-0175-y

5. Schwendicke F, Elhennawy K, Reda S, Bekes K, Manton DJ, Krois J. Global burden of molar incisor hypomineralization. J Dent. 2018 Jan;68:10-8. https://doi.org/10.1016/i.jdent.2017.12.002

6. Zhao D, Dong B, Yu D, Ren Q, Sun Y. The prevalence of molar incisor hypomineralization: evidence from 70 studies. Int J Paediatr Dent. 2018 Mar;28(2):170-9. https://doi.org/10.1111/ipd.12323

7. Oyedele TA, Folayan MO, Adekoya-Sofowora CA, Oziegbe EO. Co-morbidities associated with molarincisor hypomineralisation in 8 to 16 year old pupils in visits have implications for the psychological health of children affected with MIH. These problems highlight the need for prompt diagnosis and preventive oral care for children with MIH to reduce the risk of post-eruptive breakdown and other sequelae that may require multiple dental visits.

\section{Conclusion}

Children with MIH spend more time receiving oral health care when compared with those without $\mathrm{MIH}$. This information can help clinicians draw up a better plan for the management of children with $\mathrm{MIH}$.

\section{Acknowledgements}

We acknowledge the time and effort put in by the children and their parents in this study.

Ile-Ife, Nigeria. BMC Oral Health. 2015 Mar;15(1):37.

https://doi.org/10.1186/s12903-015-0017-7

8. Souza JF, Fragelli CM, Restrepo M, Mushashe AM, Losso EM, Cunha LF. Aesthetic management of molar incisor hypomineralisation. RSBO. 2014;11:204-8.

9. Hayes A, Azarpazhooh A, Dempster L, Ravaghi V, Quiñonez C. Time loss due to dental problems and treatment in the Canadian population: analysis of a nationwide crosssectional survey. BMC Oral Health. 2013 Apr;13(1):17. https://doi.org/10.1186/1472-6831-13-17

10. Mishu MP, Hobdell M, Khan MH, Hubbard RM, Sabbah $W$. Relationship between untreated dental caries and weight and height of 6- to 12-year-old primary school children in Bangladesh. Int J Dent. 2013;2013:629675. https://doi.org/10.1155/2013/629675

11. Jälevik B, Klingberg GA. Dental treatment, dental fear and behaviour management problems in children with severe enamel hypomineralization of their permanent first molars. Int J Paediatr Dent. 2002 Jan;12(1):24-32. https://doi.org/10.1046/i.0960-7439.2001.00318.x

12. Kotsanos N, Kaklamanos EG, Arapostathis K. Treatment management of first permanent molars in children with Molar-Incisor Hypomineralisation. Eur J Paediatr Dent. 2005 Dec;6(4):179-84.

13. 13. Temilola OD, Folayan MO, Oyedele T. The prevalence and pattern of deciduous molar hypomineralization and molar-incisor hypomineralization in children from a suburban population in Nigeria. BMC Oral Health. 2015 Jun;15(1):73. https://doi.org/10.1186/s12903-015-0059-x 
14. Oliveira MA, Bendo CB, Ferreira MC, Paiva SM, Vale MP, SerraNegra JM. Association between childhood dental experiences and dental fear among dental, psychology and mathematics undergraduates in Brazil. Int J Environ Res Public Health. 2012 Dec;9(12):4676-87. https://doi.org/10.3390/ijerph9124676

15. Olusanya $O$, Okpere $O$, Ezimokhai M. The importance of social class in voluntary fertility control in developing country. West Afr J Med. 1985;4:205-12.

16. Folayan MO, Idehen EE, Ufomata D. The effect of sociodemographic factors on dental anxiety in children seen in a suburban Nigerian hospital. Int J Paediatr Dent. 2003 Jan;13(1):20-6. https://doi.org/10.1046/j.1365-263X.2003.00411.x

17. Leppäniemi A, Lukinmaa PL, Alaluusua S. Nonfluoride hypomineralizations in the permanent first molars and their impact on the treatment need. Caries Res. 2001 JanFeb;35(1):36-40. https://doi.org/10.1159/000047428

18. Weerheijm KL, Duggal M, Mejàre I, Papagiannoulis L, Koch $G$, Martens LC et al. Judgement criteria for molar incisor hypomineralisation $(\mathrm{MIH})$ in epidemiologic studies: a summary of the European meeting on MIH held in Athens, 2003. Eur J Paediatr Dent. 2003 Sep;4(3):110-3.

19. Ligali TO, Folayan MO, Sheiham A. Assessment of time taken to treat dental trauma in Nigerian children.
Eur Arch Paediatr Dent. 2011 Feb;12(1):37-40. https://doi.org/10.1007/BF03262777

20. Kopperud SE, Pedersen CG, Espelid I. Treatment decisions on Molar-Incisor Hypomineralization (MIH) by Norwegian dentists: a questionnaire study. BMC Oral Health. 2016 Jul;17(1):3. https://doi.org/10.1186/s12903-016-0237-5

21. Rodd HD, Boissonade FM. Immunocytochemical investigation of immune cells within human primary and permanent tooth pulp. Int J Paediatr Dent. 2006 Jan;16(1):2-9. https://doi.org/10.1111/j.1365-263X.2006.00682.x

22. Mahoney EK, Rohanizadeh R, Ismail FS, Kilpatrick NM, Swain MV. Mechanical properties and microstructure of hypomineralised enamel of permanent teeth. Biomaterials. 2004 Sep;25(20):5091-100. https://doi.org/10.1016/j.biomaterials.2004.02.044

23. Lygidakis NA, Wong F, Jälevik B, Vierrou AM, Alaluusua S, Espelid I. Best clinical practice guidance for clinicians dealing with children presenting with molar-incisor-hypomineralisation (MIH): an EAPD policy document. Eur Arch Paediatr Dent. 2010 Apr;1 1(2):75-81. https://doi.org/10.1007/BF03262716

24. Mejàre I, Bergman E, Grindefjord M. Hypomineralized molars and incisors of unknown origin: treatment outcome at age 18 years. Int J Paediatr Dent. 2005 Jan;15(1):20-8. https://doi.org/10.1111/j.1365-263X.2005.00599.x 